\title{
Nueva metodología de diagnóstico de fallas en rodamientos en una máquina síncrona mediante el procesamiento de señales vibro- acústicas empleando análisis de densidad de potencia
}

\section{Novel Methodology of Fault Diagnosis on Bearings in a Synchronous Machine by Processing Vibro-Acoustic Signals Using Power Spectral Density}

\author{
Medrano-Hurtado Zulma Yadira \\ Universidad Autónoma de Baja California \\ Correo:zulmamh@yahoo.com.mx \\ Pérez-Tello Carlos \\ Instituto de Ingeniería \\ Universidad Autónoma de Baja California \\ Correo:carlosperez@uabc.edu.mx
}

Gómez-Sarduy Julio

Universidad de Cienfuegos, Cuba

Correo:jgomez@ucf.edu.cu

Vera-Pérez Maximiliano

Universidad Autónoma de Baja California

Correo:mvera@uabc.edu.mx

\section{Resumen}

Se presenta una metodología novedosa para la detección de fallas en rodamientos en un generador síncrono, empleando señales de vibración capturadas a través de transductores de aceleración (acelerómetros piezoeléctricos) y acústicos (micrófonos omnidireccionales), para procesar las señales de vibración se emplea el algoritmo conocido como densidad espectral de potencia $(P S D)$. Se analizan las señales de vibración resultantes de rodamientos, sin falla y con falla artificial, respectivamente. La falla artificial consistió en una grieta producida en la jaula de un rodamiento SKF6303-2RSH. Este método permite la utilización de micrófonos para analizar la vibración de las máquinas lo que representa no tener que montar ningún tipo de transductor en la máquina como sí ocurre con la técnica convencional de detección de fallas a través de la utilización de acelerómetros, además de ser un método que puede detectar una falla en la jaula del rodamiento.

\section{Descriptores:}

- densidad espectral de potencia

- acelerómetro piezoeléctrico

- micrófonos omnidireccionales

- falla

- jaula

- rodamiento 


\begin{abstract}
This work presents a novel methodology for detecting faults in bearings in a synchronous generator, using vibration signals captured through acceleration transducer (piezoelectric accelerometers) and acoustic transducer (omnidirectional microphone), to process vibration signals using the algorithm known as power spectral density (PSD). It analyzes vibration signals resulting from bearings, without fault and with artificial fault, respectively. The artificial fault consisted in a crack produced in a cage of a SKF6303-2RSH bearing. This method allows the utilization of microphones to analyze the vibration of machines which represents not having to mount any type of transducer on the machine as if occurs with the conventional technique of fault detection through the use of accelerometers, in addition to be a method that can detect a fault in the bearing cage.
\end{abstract}

\section{Introducción}

Los generadores síncronos generan alrededor de 99\% del consumo de electricidad mundial (Planeta neutro, 2009), por su importancia en la generación eléctrica global se hace necesaria la anticipación a la ocurrencia de fallas que puedan llegar a producir problemas indeseados. Este tipo de generadores se hallan expuestos a un gran número de fallas y constituyen el equipo más costoso del sistema eléctrico de potencia (Suárez, 1998), lo que los sitúa en una posición determinada de carácter crítico. Las fallas en rodamientos es uno de los problemas más comunes en los generadores síncronos. Aproximadamente $40 \%$ (Sin et al., 2003) de las fallas en las máquinas eléctricas rotativas.

La forma convencional de análisis y diagnóstico de falla en rodamientos se basa en el empleo de registros de señales de vibración que juegan en la actualidad un papel importante en el mantenimiento predictivo para analizar su comportamiento. En términos generales, la información aportada por las vibraciones en el dominio de la frecuencia permite determinar posibles condiciones anormales en los rodamientos de los generadores síncronos. La detección temprana de fallas en rodamientos usando las señales de vibración exige el uso de técnicas apropiadas del campo de procesamiento de señales como la densidad espectral de potencia o PSD.

De este modo, se presenta la necesidad y la oportunidad de contar con una metodología distinta, pero igualmente confiable, que permita la utilización de otro tipo de señal que no sea invasiva. Teniendo como base lo anterior, los autores proponen una metodología de diagnóstico utilizando señales generadas como emisión acústica de las máquinas a través de la utilización de micrófonos omnidireccionales para la detección de fallas en rodamientos. Asimismo, se realiza un estudio comparativo utilizando técnicas de detección convencional a través de acelerómetros piezoeléctricos. La señal de vibración ocasionada por un rodamiento puede contener componentes espectrales que están relacionados con la geometría del rodamiento, el número de elementos rodantes, la velocidad de rotación, la ubicación de la falla y el tipo de carga aplicada.

Cualquiera que sea la causa de la vibración en un generador síncrono esta resulta en una emisión acústica. El uso de tales señales permiten contar con un sistema y una metodología flexibles que pueden utilizarse en varios equipos y máquinas de manera frecuente, debido a la facilidad de instalación, montaje, desmontaje y transportación que representa este tipo de dispositivos al no tener que permanecer de manera permanente dentro de la máquina de manera única.

Algunas características y ventajas dentro de la emisión acústica son:

1. La emisión acústica detecta el movimiento, lo que permite la detección temprana de fallas, así como su evolución en el tiempo.

2. Es una prueba global debido a que esta metodología puede llegar a utilizarse en muchas de las máquinas presentes en el sistema.

3. No es invasiva, ya que no se requiere montaje de transductores o medidores directamente sobre la máquina, no interfiriendo con su funcionamiento normal.

4. Las fallas crean su propio patrón de señal de emisión acústica, por lo que cada condición es única (para cada máquina, y para cada falla).

5. Es una prueba relativamente rápida.

6. Las pruebas son fácilmente repetibles.

La principal desventaja dentro de la emisión acústica es que la prueba es sensible al ruido ambiental de fondo, 
el cual puede compensarse a través de la utilización de un supresor de ruido para un detector de fallo Noise Cancellation Fault Detector (NCFD), que permiten cancelar el ruido ambiental de fondo en donde se encuentran ubicadas las máquinas eléctricas que se desean monitorear sin afectar la emisión acústica relacionada con la falla (Detectando fallas en rodamientos utilizando métodos de prueba eléctricos y mecánicos de vibración, 2009).

\section{Causas de fallas en los rodamientos}

Entre las causas más probables de fallas en rodamientos se encuentran distribuidas: $36 \%$ del resultado de una lubricación inadecuada; $34 \%$ resultado de una operación inadecuada como pudiera ser: cargas dinámicas excesivas sobre el rodamiento, producto de una falla de diseño de la máquina o de la presencia de fuerzas externas como desbalance y desalineamiento; $14 \%$ se debe a la contaminación, incluyendo humedad; y cerca de 16\% son defectos originados en el proceso de montaje (mal manejo, causado por el almacenamiento, transportación e instalación inadecuada del rodamiento) (Acoustic Fault Detection of Mechanical Systems with active noise cancellation, 2013). En la mayoría de los casos, las fallas no aparecen de manera inesperada sino gradualmente, lo que hace posible su detección antes de que las consecuencias resulten catastróficas.

\section{Etapas de fallas de rodamientos}

En una investigación desarrollada por fabricantes, se determinó que los rodamientos operan aproximadamente $80 \%$ de su vida útil libres de defectos y cuando una falla ocurre, generalmente se encuentran en alguna de las cuatro diferentes etapas de falla (Acoustic Fault Detection of Mechanical Systems with active noise cancellation, 2013):

Etapa 1: Se tiene una vibración con componentes de alta frecuencia (generalmente mayores a $5 \mathrm{kHz}$ ), producto de la generación de ondas de esfuerzo y de otros tipos, las que a su vez, excitan frecuencias naturales del soporte y las pistas del rodamiento, así como del sensor con el cual se realiza la medición. En esta etapa la temperatura no se incrementa y las grietas no son visibles y con frecuencia ocurren debajo de la superficie de las pistas. En esta etapa aún no es necesario el cambio del rodamiento.

Etapa 2: Las grietas empiezan a ser visibles al ojo humano y el rodamiento presenta emisión acústica, además de que la temperatura en algunos casos se incrementa. En esta etapa aparecen componentes frecuenciales relacionadas con las frecuencias de falla de los rodamientos en la zona de frecuencia baja e intermedia y hay un incremento de ruido audible.

Etapa 3: Conforme la grieta avanza, pueden aparecer gran cantidad de bandas laterales alrededor de las frecuencias de falla relacionadas con la velocidad de rotación y modulación entre las diferentes frecuencias de falla cuando la grieta se ha extendido a pistas y elementos rodantes. En esta etapa se hace necesario comenzar a programar el cambio del rodamiento.

Etapa 4: Se encuentra cerca de una falla catastrófica, la emisión acústica aumenta significativamente y se produce sobrecalentamiento. En esta etapa, la vibración en la región de alta frecuencia disminuye, crece la componente a la velocidad de rotación y las vibraciones tienden a ser aleatorias. En este momento, será necesario el cambio inmediato del rodamiento.

\section{Marco teórico}

\section{Análisis de vibración}

Bajo condiciones normales de funcionamiento, los rodamientos fallan por desgaste o fatiga del material, cuando comienzan a fallar se incrementan las vibraciones de los generadores síncronos y los niveles de emisión acústica aumentan. Estas frecuencias de falla de rodamientos se encuentran en función de la geometría de los rodamientos y la velocidad de marcha (Averías de los rodamientos. Reconociemiento de daños e inspección de rodamientos, 2003; NTN SNR, análisis vibratorio, 2004; SKF, fallos en los rodamientos y sus causas; Villada et al., 2012; Stack et al., 2004; Palomino, 2011; Wowk, 1991; Batkov y Barkova, 1999). Cada uno de los componentes del rodamiento presenta una frecuencia de falla única y se definen en al menos cuatro frecuencias características que se producen en los rodamientos cuando la falla se produce en la pista exterior, Ball Pass Frequency of Theouterrace (BPFO) en la pista interior, Ball Pass Frequency of the Inner Race (BPFI), en los elementos rodantes, Ball Spin Frequency (BSF), en la jaula, Fundamental Train Frecuency (FTF):

$$
\begin{aligned}
& B P F O=\frac{n e}{2} \cdot \frac{V_{r p m}}{60} \cdot\left[1-\frac{d}{D} \cdot \cos (\beta)\right] \\
& B P F I=\frac{n e}{2} \cdot \frac{V_{r p m}}{60} \cdot\left[1+\frac{d}{D} \cdot \cos (\beta)\right] \\
& B S F=\frac{D}{d} \cdot \frac{V_{r p m}}{60} \cdot\left[1-\left(\frac{d}{D}\right)^{2} \cdot \cos ^{2}(\beta)\right]
\end{aligned}
$$


$F T F=\frac{V_{r p m}}{120} \cdot\left[1-\frac{d}{D} \cdot \cos (\beta)\right]$

donde

$D$ = diámetro de paso $(\mathrm{mm})$

$d$ = diámetro de bolas $(\mathrm{mm})$

$\beta$ = ángulo de contacto entre las bolas y las pistas

$V_{r p m}=$ velocidad de rotación ( $\mathrm{rpm}$ )

ne $=$ número de elementos rodantes

Las frecuencias de falla son puntos de referencia al analizar el espectro obtenido de las señales de vibración de los rodamientos. Si en un rodamiento existe un problema, el espectro proporciona información para ayudar a determinar la localización del problema, la causa del problema, la tendencia y hasta qué tanto llega a ser crítico (Suárez, 1998; Averías de los rodamientos. Reconociemiento de daños e inspección de rodamientos, 2003).

La vibración es la oscilación de un cuerpo alrededor de un punto de reposo y existen dos conceptos relacionados con ella: la frecuencia y la amplitud. Estas oscilaciones pueden producirse por:

- Desequilibrio en el acoplamiento del sistema motorgenerador.

- Falla de algún elemento en el generador síncrono.

\section{Densidad espectral de potencia (PSD)}

El análisis de señales en el dominio de la frecuencia permite descubrir aspectos de la señal que son muy difíciles o imposibles de observar a partir de su representación en el dominio del tiempo. Cuando estas componentes se grafican en el dominio de la frecuencia se detectan fácil debido a que no quedan enmascaradas por señales de gran amplitud.

Un truncamiento o ventaneo (windowing) en el dominio del tiempo, limita la longitud de una señal a un número de muestras $\mathrm{N}$ arbitrario, lo que permite el conocimiento de la señal completa.

Por ejemplo, si se analiza una señal de longitud infinita compuesta por una sinusoide

$x(t)=A \sin (\omega t+\theta),-\infty<t<+\infty$

donde

$A$ = amplitud

$\omega=$ frecuencia en $\mathrm{rad} / \mathrm{seg}$

$\theta$ = ángulo de fase en rad
Al tomar parte de la señal $x(t)$, con una longitud de $N$ muestras enteras comprendidas en el intervalo $0 \leq n \leq N-1$, permite definir una nueva señal que se denota como $x(n)$. Se puede pensar en esta señal como el resultado de multiplicar la señal original $x(t)$ por una ventana $w[n]$.

$x(n)=x(t) w[n]$

La más simple de las medidas de energía (o potencia) en una señal en función de frecuencia es la densidad espectral de energía, que es solo la amplitud del espectro, al cuadrado. En términos de los coeficientes de Fourier $\left\{c_{k}\right\}$

$\left|c_{k}\right|^{2}=c_{k} \cdot c_{k}^{*}$

donde $c k=\frac{1}{N} \sum_{n=0}^{N-1} x(n) e^{-j 2 \pi k n / N}$

La energía de una señal periódica en tiempo discreto con periodo $N$ se define como

$E_{N}=\sum_{n=0}^{N-1}|x(n)|^{2}=N \sum_{k=0}^{N-1}\left|c_{k}\right|^{2}$

El promedio de la potencia de la señal en un intervalo $T$ es simplemente la energía normalizada por la longitud de observación

$P_{x}=\frac{1}{T}|x(n)|^{2}=\frac{1}{N} \sum_{n=0}^{N-1}|x(n)|^{2}$

A medida que el intervalo de observación T se hace grande, este converge en la verdadera potencia de la señal

$P=\lim _{T \rightarrow \infty} P_{x}$

Derivando una expresión para $P_{x}$

$$
\begin{aligned}
& P_{x}=\frac{1}{N} \sum_{n=0}^{N-1} x(n) x^{*}(n)=\frac{1}{N} \sum_{n=0}^{N-1} x(n)\left(\sum_{n=0}^{N-1} c_{k}^{*} e^{-j 2 \pi k n / N}\right) \\
& P_{x}=\sum_{n=0}^{N-1} c_{k}^{*}\left[\frac{1}{N} \sum_{n=0}^{N-1} x(n) e^{-j 2 \pi k n / N}\right]=\sum_{k=0}^{N-1}\left|c_{k}\right|^{2} \\
& P_{x}=\frac{1}{N} \sum_{n=0}^{N-1}|x(n)|^{2}
\end{aligned}
$$


Que es la expresión deseada para la potencia media de una señal periódica, que es la suma de las potencias medias de las componentes individuales en frecuencia. La secuencia $\left|c_{k}\right|^{2}$ para $k=0,1, \ldots N T-1$ es la distribución de potencia en función de la frecuencia y se denomina densidad espectral de potencia (PSD) de una señal periódica.

La PSD se define como

$$
\operatorname{PSD}(x(n))=\sum_{k=0}^{N-1}\left|c_{k}\right|^{2}, k=0,1, \ldots, N-1
$$

Si se grafica $\left|c_{k}\right|^{2}$ en función de las frecuencias $k F_{0}$ para $k=0, \pm 1, \pm 2, \ldots$, el diagrama obtenido muestra como se distribuye la potencia de la señal periódica entre sus distintas componentes en frecuencia. Este diagrama, es el PSD de la señal periódica $x(n)$. Dado que la potencia de una señal periódica existe solo para determinados valores discretos de frecuencia (es decir, $F=0, \pm F_{0}$, $\left.\pm 2 F_{0}, \ldots\right)$, se dice que la señal tiene un espectro formado por líneas. El espaciado entre dos líneas espectrales consecutivas es igual al inverso del periodo fundamental $T_{p}$, mientras que la forma del espectro (es decir, la distribución de potencia de la señal), depende de las características de la señal en el dominio del tiempo.

La PSD deberá ser una función real, simétrica en $f$ para $x(n)$ real. Por esta razón, el espectro de potencia de las funciones reales se traza normalmente solo para frecuencias positivas. Debido a que no se pueden hacer cálculos en una señal de longitud infinita, todas las PSD en la práctica son estimaciones de $P_{x}$

$$
\begin{aligned}
& P S D_{x}(\omega)=\sum_{n=-\infty}^{+\infty} x(n) e^{\{-j(2 \pi f n)\}} \\
& P S D_{x}(\omega)=\sum_{n=-\infty}^{+\infty} x(n) e^{\{-j(\omega n)\}}
\end{aligned}
$$

donde $w=2 \pi f$

La forma más simple para estimar la PSD es truncar los datos con una ventana rectangular de $N$ muestras de longitud, al cual se le denomina periodograma. Se introdujo originalmente por Schuster (1898) para detectar y medir periodicidades escondidas en los datos (Nikias y Raughuveer, 1987; Toledo et al., 2001). Acotando a $N$ muestras se tiene

$$
P S D_{x}(\omega)=\frac{1}{N} \sum_{m=0}^{N-1} \sum_{i=0}^{N-1} x(i) x(i+m) e^{\left\{-j\left(\omega \frac{m}{N}\right)\right\}}
$$

Por tanto, el periodograma se escribe

$$
P S D_{x}(k)=\frac{1}{N} X(k) X^{*}(k)
$$

$P S D_{x}(k)=\frac{1}{N}|X(k)|^{2}$

donde $\mathrm{X}(k)=\sum_{n=-\infty}^{\infty} x(n) e^{-j 2 \pi f n} \quad$ es la transformada de Fourier de tiempo discreto de la secuencia de datos de $N$ puntos.

El periodograma así modificado tiene por objetivo solucionar el problema de enmascaramiento espectral producido por el periodograma ocasionado por la gran amplitud de los lóbulos secundarios de la ventana rectangular. Para ello, se propone desplegar el proceso con una ventana general $(w[n]=1)$. Welch, en 1967 hizo dos modificaciones básicas al método de Bartlett (1948) (Proakis y Manolakis, 1998).

La primera modificación permitió el solapamiento (overlap) de segmentos de datos. Suponiendo que entre dos secuencias consecutivas existe un desplazamiento de $D$ puntos y que cada secuencia consta de $L$ puntos de longitud, la secuencia $i$-ésima viene determinada por la expresión

$$
x i(n)=x(n+i D), \begin{aligned}
n & =0,1, \ldots, M-1 \\
i & =0,1, \ldots, L-1
\end{aligned}
$$

donde $i \mathrm{D}$ es el punto de inicio de la secuencia $i$-ésima. El solapamiento entre dos frecuencias consecutivas $x_{i}(n)$ y $x_{i+1}(n)$ es de $L-D$ puntos, y si las $K$ secuencias cubren una longitud de $N$ puntos, entonces

$N=L+(K-1)$

Suponiendo que no existe solapamiento entre las secuencias $(D=L)$; tenemos $K=N / L$ secciones de longitud $L$, como en el método de Bartlett.

De esta manera se mantiene la resolución (la longitud no varía) del método de Bartlett. Con lo que se mejora la resolución manteniendo la misma varianza que en el método de Bartlett.

La segunda modificación consiste en enventanar cada secuencia $x_{i}(n)$ con una ventana general $w[n]$, antes de calcular el periodograma. De esta manera se obtiene el periodograma modificado para cada secuencia enventanada

$\tilde{P}_{x x}^{(i)}(k)=\frac{1}{L U}\left|\sum_{n=0}^{L-1} x_{i}(n) w[n] e^{-j w n}\right|^{2}$

donde $U$ es un factor de normalización para la potencia de la función ventana y se selecciona como

$U=\frac{1}{L} \sum_{n=0}^{L-1}|w[n]|^{2}$ 
El espectro mediante el periodograma modificado puede expresarse como

$\tilde{P}_{x x}^{(i)}(k)=\frac{1}{U} E\left[c_{k}(k) c_{k}^{*}(k)\right]$

El símbolo $E$ se usa como operador en las variables aleatorias. Se llama esperanza, valor esperado o valor promedio.

donde $c_{k}=\sum_{n=0}^{N-1} x(n) w\left[\frac{n}{A}\right] e^{-j 2 \pi k / N}$

El parámetro $A$ determina el ancho de la ventana y es un entero dependiente del tamaño de la sección $N$. Se recomienda un método empírico (Nikias y Raughuveer, 1987; Proakis y Manolakis, 1998). para la elección del parámetro $A$. La ventana utilizada en este trabajo fue la rectangular.

\section{Metodología experimental}

Se puede observar en la figura 1, la metodología convencional para la detección de fallas en rodamientos en generadores síncronos, (cuadros con líneas continuas), así como los pasos para evaluar el desempeño del PSD (cuadros con línea punteada) con los transductores acústicos:

1. Determinación del tipo de máquina a analizar (generador síncrono).

2. Determinación del tipo de falla a analizar (falla en rodamiento).
3. Determinación de los transductores a utilizar para la captura de señales de vibración (acelerómetros y micrófonos).

4. Conocer las características de los transductores a utilizar (acelerómetros triaxiales piezoeléctricos y micrófonos omnidireccionales).

5. Conocer las características de geometría del rodamiento a analizar.

6. Conocer los tipos de fallas en rodamientos (determinación de las frecuencias de falla del rodamiento).

7. Determinación del tipo de falla a analizar (grieta en la jaula del rodamiento).

8. Diseño y construcción del banco de pruebas.

9. Adquisición de señales de vibración a través de los acelerómetros piezoeléctricos y los micrófonos omnidireccionales (en condiciones normales de operación y con falla artificial).

10. Análisis de las señales a través del método de procesamiento de señal (PSD).

11. Relación entre fallas y PSD.

12. Obtención de conclusiones.

\section{Ensayos y resultados}

Se llevaron a cabo ensayos con rodamientos sin falla y con grieta en la jaula de un rodamiento SKF6303 $2 R S H$. Las pruebas se realizaron en un banco de pruebas como se puede observar en la figura 2, cuyas características se indican en la tabla 1. Para el análisis de vibraciones se utilizaron transductores de aceleración (acelerómetros piezoeléctricos) y transductores acústicos (micrófonos omnidireccionales).

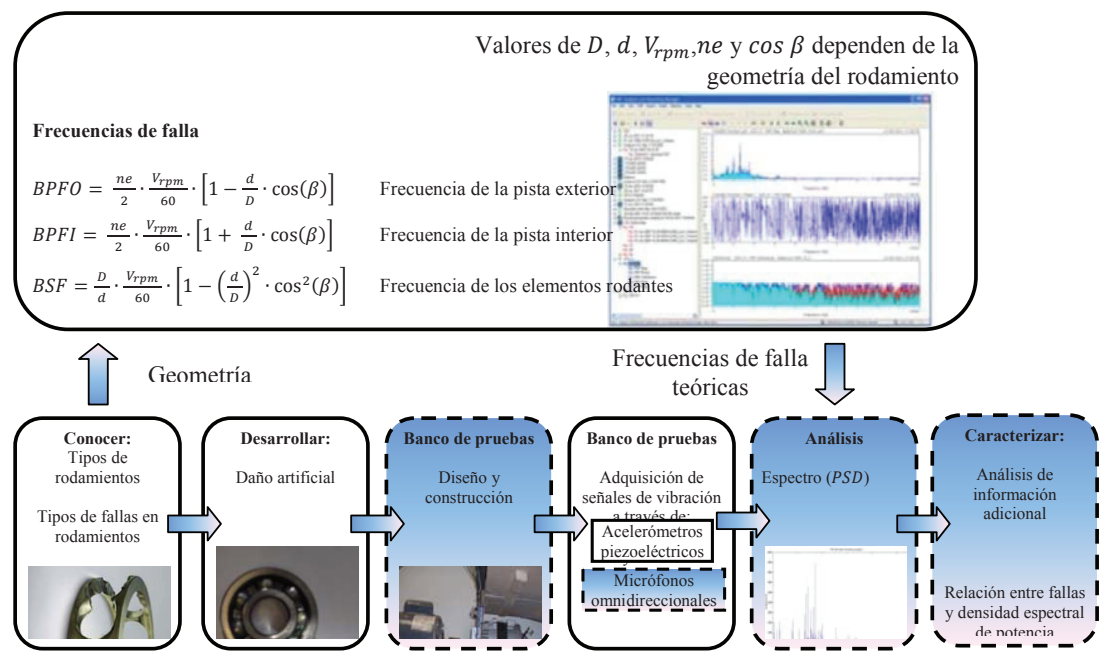

Figura 1. Metodología de la experimentación (Capistrán y Paredes, 2005) 

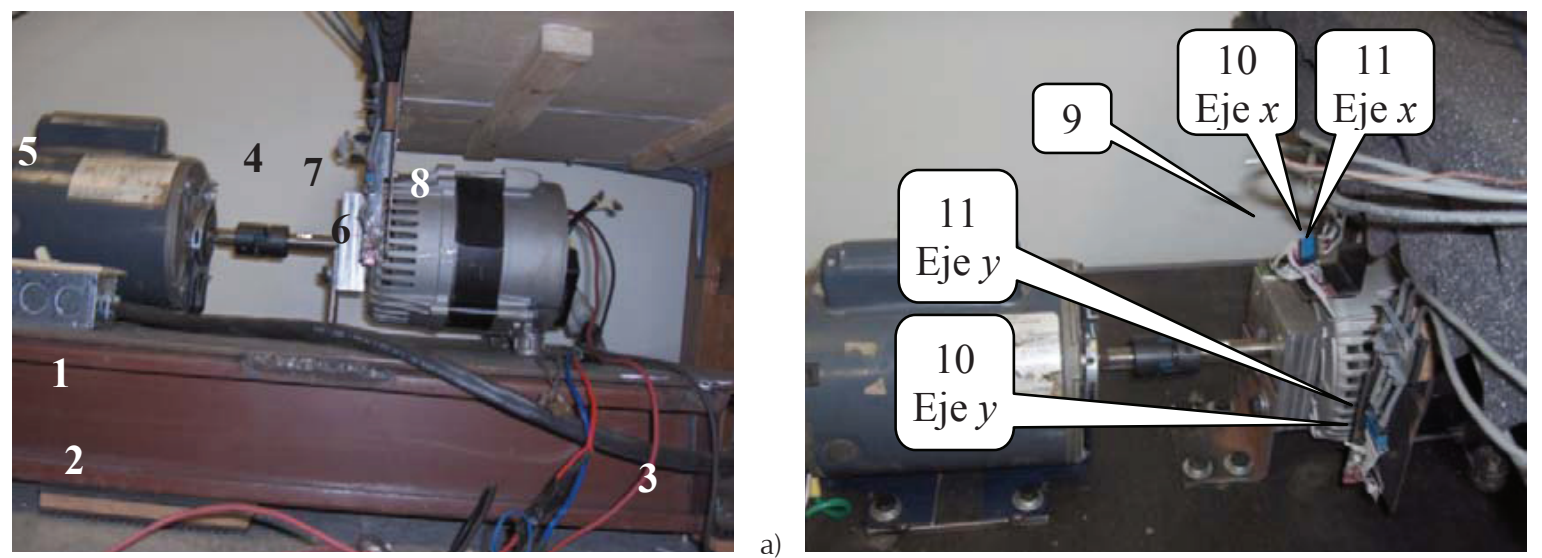

b)

Figura 2. Sistema mecánico: a) banco experimental, b) colocación de los transductores

Tabla 1. Características del banco de prueba

\begin{tabular}{|c|c|c|}
\hline Núm. & Cant. & Descripción \\
\hline 1 & 1 & Base (plancha de metal $6.35 \times 10^{-3} \mathrm{~m}$, polín calibre 12) \\
\hline 2 & 4 & Amortiguadores de corcho y neopreno \\
\hline 3 & 4 & Tornillos roscables \\
\hline 4 & 1 & Acoplamiento elástico lovejoy de $12.7 \times 10^{-3}$ a $12.7 \times 10^{-3} \mathrm{~m}$ \\
\hline 5 & 1 & Motor de inducción monofásico GE, CATNO.C 1158,1 Hp, $60 \mathrm{~Hz}, 115 / 208$ - 230 V, 1725 rpm, 14.7 A \\
\hline 6 & 1 & Base de aluminio de $(0.1 \times 0.1 \times 0.03 \mathrm{~m})$ \\
\hline 7 & 2 & rodamiento SKF6303-2RSH \\
\hline 8 & 1 & Generador síncrono, $2 \mathrm{Hp}, 50 \mathrm{Vcd}$ \\
\hline 9 & 1 & Acelerómetro 3 ejes (Analog Device $E V A L-A D X L 325$ ) \\
\hline 10 & 2 & Micrófono omnidireccional (Panasonic $W M-61 A$ ) \\
\hline 11 & 2 & Micrófono unidireccional (Panasonic WM - 55A103) \\
\hline
\end{tabular}

\section{Frecuencias de falla del rodamiento}

El deterioro en alguno de los componentes del rodamiento produce un pico a cierta frecuencia, el valor de esta frecuencia depende de la geometría del rodamiento, así como de su velocidad de rotación (Averías de los rodamientos. Reconocimiento de daños e inspección de rodamientos, 2003; Palomino, 2011; SKF). Las características de rodamiento para el cálculo de las frecuencias de vibración de las ecuaciones 1 a 4 son (NTN SNR, 2004; SKF):

$D=32 \mathrm{~mm} \quad d=8.731 \mathrm{~mm} \quad \beta=0^{\circ}$ ne $=7$ bolas $V_{r p m}=1725 \mathrm{rpm}$ (revoluciones de operación del impulsor).

Se pueden observar en la tabla 2 los valores de las frecuencias de vibración para los elementos que componen el rodamiento.

Tabla 2. Frecuencias de falla para los elementos del rodamiento SKF6303-2RSH

\begin{tabular}{|c|c|c|c|c|c|}
\hline \multicolumn{2}{|c|}{ Frecuencia de rotación } & \multicolumn{4}{|c|}{$\begin{array}{l}\text { Frecuencias } \\
\qquad(\mathrm{Hz})\end{array}$} \\
\hline & & $\begin{array}{l}\text { Defecto en la pista } \\
\text { interior }\end{array}$ & $\begin{array}{l}\text { Defectos en la pista } \\
\text { exterior }\end{array}$ & Defectos en la jaula & $\begin{array}{l}\text { Defectos en los elementos } \\
\text { rodantes }\end{array}$ \\
\hline 60 & 1725 & 128.080 & 73.170 & 10.453 & 97.527 \\
\hline
\end{tabular}


Las señales de vibración y acústicas del rodamiento se adquirieron a una tasa de 6000 muestras/segundo, con una tarjeta para adquisición de datos NI USB - 6009, tomando una muestra representativa de 6000 muestras evitando los transitorios del arranque registrando la muestra después de cinco minutos de operación.

Análisis de la señal en el dominio de la frecuencia (acelerómetros y micrófonos)

La figura 3 muestra los resultados obtenidos para la señal horizontal (eje $x$ ) del acelerómetro piezoeléctrico para el generador, graficada en amplitud vs frecuencia: a) $\sin$ falla y b) con falla.
La figura 4 muestra los resultados para la señal vertical (eje $y$ ) del acelerómetro piezoeléctrico para el generador, graficada en amplitud $v$ f frecuencia: a) sin falla y b) con falla.

La figura 5 muestra los resultados para la señal eje $x$ acústica del micrófono omnidireccional para el generador, graficada en amplitud $v$ s frecuencia: a) $\sin$ falla y b) con falla.

La figura 6 muestra los resultados para la señal eje $y$ acústica del micrófono omnidireccional para el generador, graficada en amplitud $v$ f frecuencia: a) sin falla y b) con falla.

En Ypma y Upma (1997) se explica el efecto de traslape en el uso de la PSD. Se hizo el análisis de las gráficas analizadas para una frecuencia de $682 \mathrm{~Hz}$, que
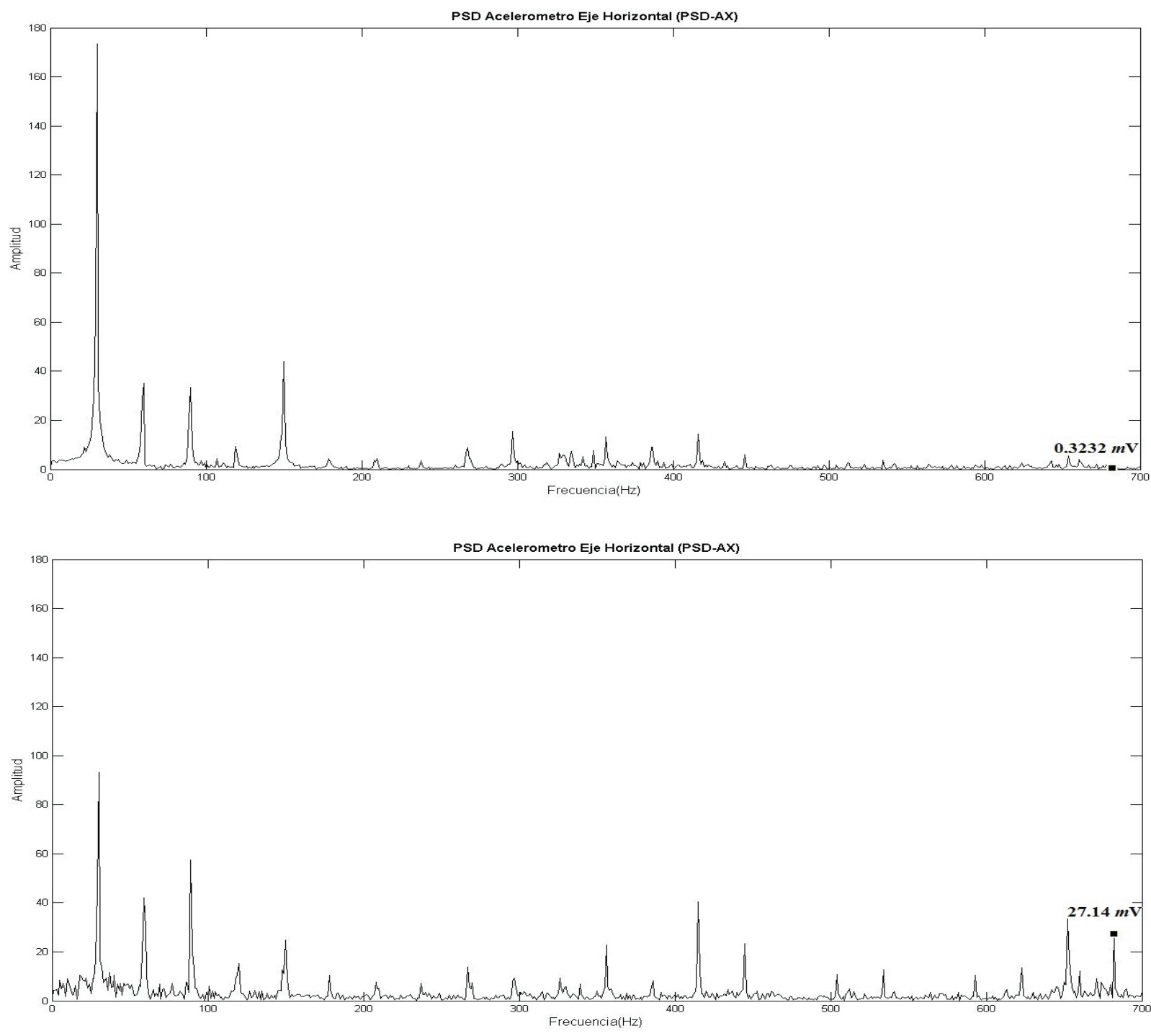

Figura 3. Señal de vibración eje $x$ del acelerómetro piezoeléctrico para la condición: a) sin falla y b) con falla 

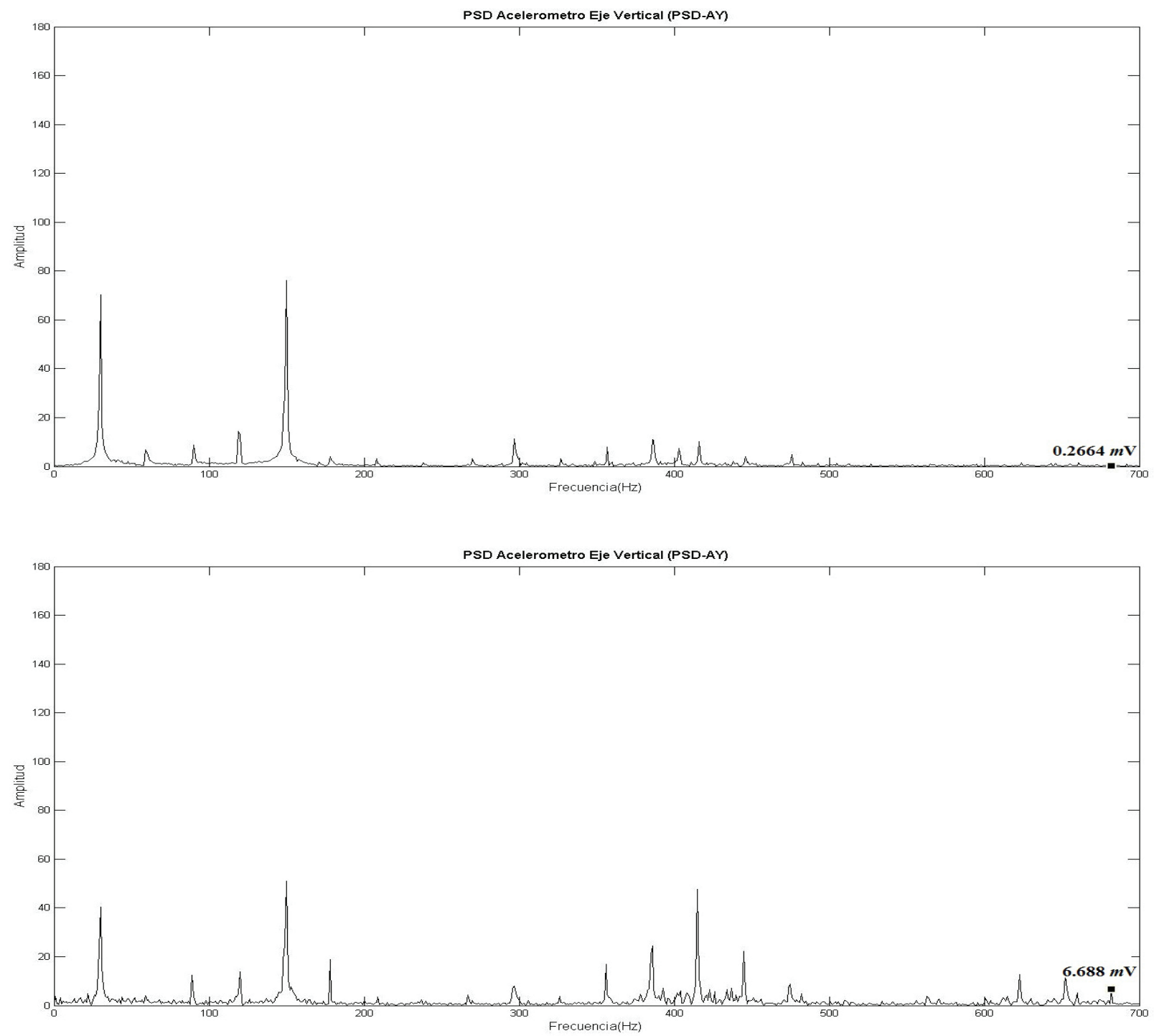

Figura 4. Señal de vibración eje y del acelerómetro piezoeléctrico para la condición: a) sin falla y b) con falla

representa aproximadamente 23 veces la frecuencia de rotación de la flecha.

En la figura 3 se muestran las gráficas de la señal del acelerómetro piezoeléctrico en el eje $x$ graficada en amplitud $v$ s frecuencia, respecto a: a) la condición sin falla, la amplitud es de $0.3232 \mathrm{mV} / \mathrm{Hz}, \mathrm{b}$ ) la condición con falla, la amplitud es de $27.14 \mathrm{mV} / \mathrm{Hz}$.

En la figura 4 se muestran las gráficas de la señal del acelerómetro piezoeléctrico en el eje $y$ graficada en amplitud vs frecuencia respecto a: a) la condición sin falla, la amplitud es de $0.2664 \mathrm{mV} / \mathrm{Hz}$, b) la condición con falla, la amplitud es de $6.688 \mathrm{mV} / \mathrm{Hz}$.

En la figura 5 se muestran las gráficas de la señal del micrófono omnidireccional en el eje $x$ graficada en amplitud vs frecuencia respecto a: a) la condición sin falla, la amplitud es de $20.28 \mathrm{mV} / \mathrm{Hz}$, b) la condición con falla, la amplitud es de $2941 \mathrm{mV} / \mathrm{Hz}$.

En la figura 6 se muestran las gráficas de la señal del micrófono omnidireccional en el eje $y$ graficada en amplitud vs frecuencia respecto a: a) la condición sin falla, la amplitud es de $14.26 \mathrm{mV} / \mathrm{Hz}$, b) la condición con falla, la amplitud es de $2148 \mathrm{mV} / \mathrm{Hz}$.

Cuando la falla es grave aparecen bandas laterales alrededor de las frecuencias importantes de rotación de la máquina. En las figuras 3, 4, 5 y 6 se analizaron los picos de frecuencia de 29.5, 59.5, 89 y $118.5 \mathrm{~Hz}$. Se observó que estas frecuencias se relacionan con la frecuencia de rotación de la flecha, ya que la relación de cada una de estas frecuencias es de $30 \mathrm{~Hz}$ aproximadamente (que es el valor teórico de rotación de la flecha). 

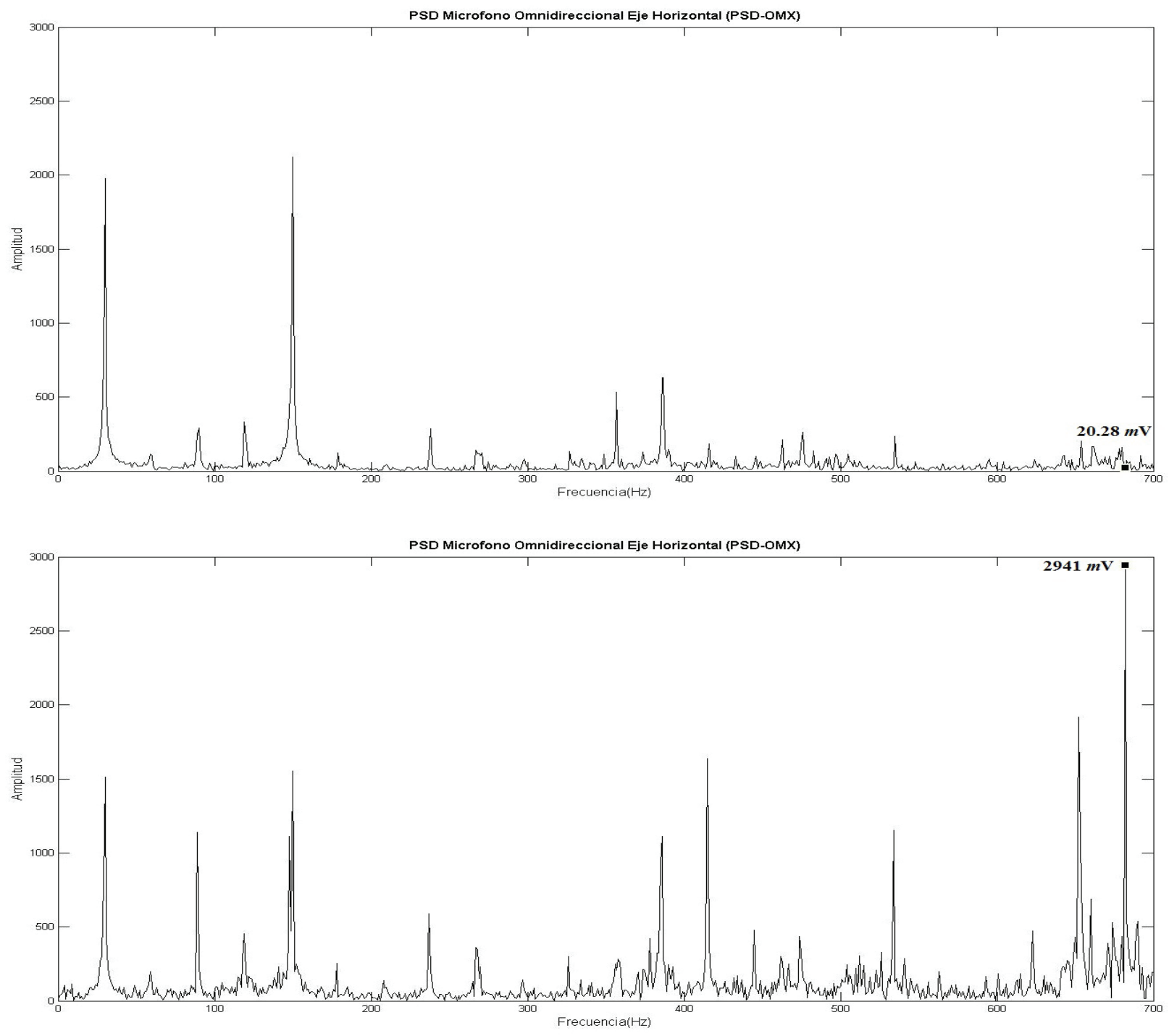

Figura 5. Señal acústica eje $x$ del micrófono omnidireccional para la condición: a) sin falla y b) con falla

Cabe señalar que cuando la falla es en la jaula las frecuencias de falla decaen sin llegar a formar bandas laterales alrededor de las frecuencias de falla. Esto impide realizar un diagnóstico para la falla en la jaula en una fase de deterioro, en el rodamiento, a través de la utilización de acelerómetros.

Cuando la falla se encuentra en la etapa 4 (etapa final del rodamiento), las frecuencias de falla disminuyen o desaparecen y surgen muchas componentes a frecuencias aleatorias presentándose un aumento en el ruido de fondo (Curso de análisis de vibraciones, 2013; Cano, 2011).

La $P S D$, es la principal técnica de procesamiento que se utiliza en el diagnóstico de máquinas debido a que permite detectar señales de alta y baja frecuencia.

\section{Discusión y análisis de resultados}

Resultados de las señales graficadas respecto a la frecuencia $(P S D)$

1.- Los picos de frecuencia que se presentan en las PSD's de las señales del acelerómetro piezoeléctrico en los ejes $x$ e $y$ para la condición sin falla son los relacionados a la frecuencia de giro del eje de la máquina (aproximadamente $30 \mathrm{~Hz}$ )

2.- Los picos que se presentan en las $P S D^{\prime} s$ de las señales del acelerómetro piezoeléctrico en los ejes $x$ e y para la condición con falla son los relacionados con la frecuencia de giro del eje de la máquina (aproximadamente $30 \mathrm{~Hz}$ ), la frecuencia de falla para la jaula del 

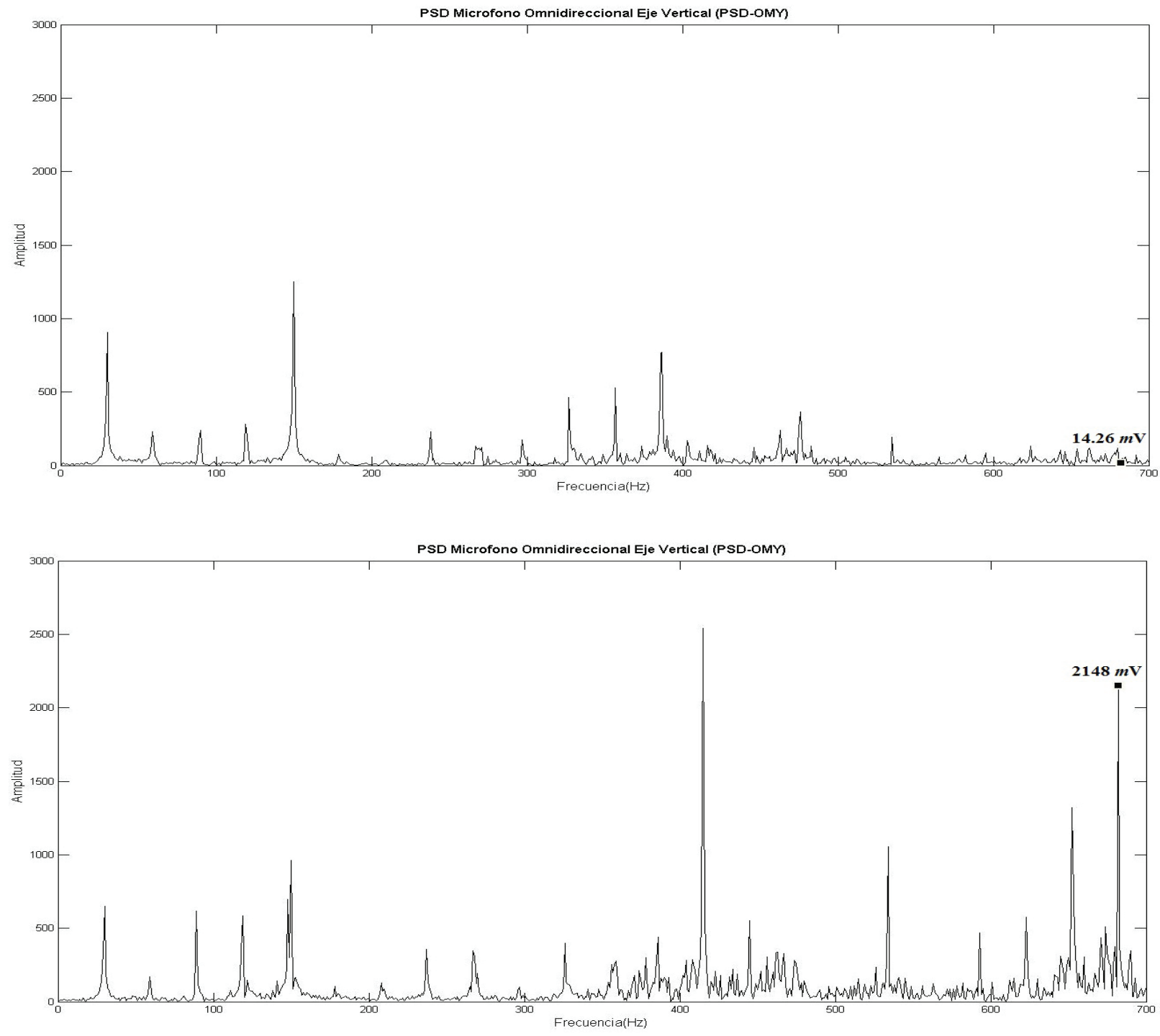

Figura 6. Señal acústica eje y del micrófono omnidireccional para la condición: a) sin falla y b) con falla

rodamiento (aproximadamente $11 \mathrm{~Hz}$ ) se encuentra enmascarada por otras frecuencias de operación de la máquina, lo que hace complicado poder tomar un dictamen de diagnóstico con este tipo de gráficas.

3.- Los picos que se presentan en las $P S D^{\prime}$ 's de las señales acústicas de los micrófonos omnidireccionales en los ejes $x$ e $y$ para la condición sin falla es menor respecto a la condición con falla, los picos de frecuencia relacionados con la frecuencia de giro del eje de la máquina (aproximadamente $30 \mathrm{~Hz}$ ).

4.- Los picos que se presentan en las $P S D^{\prime}$ 's de las señales acústicas de los micrófonos omnidireccionales en los ejes $x$ e $y$ para la condición con falla es la relacionada con la frecuencia de falla para la jaula del rodamiento (aproximadamente $11 \mathrm{~Hz}$ ), lo que permite tomar un dictamen de diagnóstico en la máquina, ya que este tipo de gráfica proporciona información del origen de la falla.

\section{Conclusiones}

Para la fase de experimentación se utilizó un sistema convencional de adquisición, el cual consistió de acelerómetros piezoeléctricos triaxiales, micrófonos omnidireccionales en los ejes $x$ e $y$. Del análisis de los resultados se puede deducir que:

1. Los acelerómetros piezoeléctricos no detectan diferencias significativas para las condiciones sin falla y con falla, por lo que se concluye que este tipo de trans- 
ductores no son una opción para el objetivo del trabajo, que es buscar un método de medición confiable de detección de falla por análisis de señales de vibración.

2. Los micrófonos omnidireccionales demuestran que son capaces de detectar una falla en la jaula del rodamiento de un generador síncrono tanto en la dirección $x$ como en la dirección $y$ a partir de las señales acústicas emitidas por esta.

3. Por tanto, se concluye que el uso de micrófonos omnidireccionales es una opción viable para detectar falla de rodamiento. Entonces el análisis de señales se puede realizar a través de la PSD y para convertir estas señales en una variable cuantitativa se puede compar y analizar contra aquellas obtenidas bajo condiciones normales de operación. De esta forma se puede dar seguimiento a la evolución del espectro de señales de una máquina, con lo que fácilmente puede detectarse cualquier señal anómala o cambio que pudiera indicar, con antelación, una posible falla.

4. El incremento en amplitud del nivel acústico es un parámetro que permitió detectar el daño en el rodamiento, mientras que la serie de picos separados por la frecuencia de falla permitió determinar la ubicación del daño en la jaula. Se comprobó que los micrófonos omidireccionales, pueden aplicarse en el monitoreo de máquinas.

\section{Innovación científica}

Es una metodología que utiliza técnica de procesamiento de señal PSD para analizar las señales acústicas de los generadores síncronos, lo que permite identificar fallas tempranas en sus rodamientos y establecer diagnósticos acertados y confiables con menor invasividad que los obtenidos por los métodos empleados hasta el momento.

\section{Referencias}

Acoustic Fault Detection of Mechanical Systems with Active Noise Cancellation. Patent application number: 20130182865, 2013 [en línea] [Fecha de consulta: 15 de febrero de 2015]. Disponible en: http://www.faqs.org/patents/app/20130182865\#ixzz 3SDtPXKxL

Averías de los rodamientos. Reconocimiento de daños e inspección de rodamientos. Núm de publ. WL 82 102/2 SB. Barcelona, 2003 [en línea] [Fecha de consulta: 20 de abril de 2014]. Disponible en: http://www.rodaunion.com/descargas/catalogos/pdf/roda mientos_y_accesorios/fag/Averias_de_Rodamientos_ WL82102-2_SB_0502.pdf
Batkov A.V., Barkova N.A. The artificial intelligence systems for machine condition monitoring and diagnostics by vibration, Proceedings of the Saint Petersburg Post Graduate Institute of the Russian Federation Power Industry and Vibration Institute, volumen 9, 1999 [en línea] [Fecha de consulta: 08 de marzo de 2014]. Disponible en: http://www.vibrotek.com/article.php? article=articles/intelect-eng/index.htm

Cano A. Diseño de aplicación informática para docencia en diagnosis de defectos en rodamientos, tesis (licenciatura), Universidad Carlos III de Madrid, 2011, 350 p.

Capistrán V. y Paredes R. Diagnóstico de condiciones de operación de rodamientos, en máquinas usando espectros de orden superior, tesis (maestría), Cuernavaca, México, Cenidet, 2005, 205 p.

Curso de análisis de vibraciones. Fases de deterioro en rodamientos, 2013 [en línea] [Fecha de consulta: 05 de febrero de 2014]. Disponible en: http://www.sinais.es/Recursos/Curso-vibraciones/rodamientos/deterioro_rodamientos.html

Detectando fallas en rodamientos utilizando métodos de prueba eléctricos y mecánicos de vibración, 2009 [en línea] [Fecha de consulta: 25 de mayo de 2014]. Disponible en: http://confiabilidad.net/assets/ uploads/art/PDF/detectando_fallas_en_rodamientos_atp.pdf

Jason R.S., Ronald G.H., Thomas G.H. An amplitude modulation detector for fault diagnosis in rolling element bearing. IEEE Transactions on Industrial Electronics, volumen 51, 2004: 10971102.

Nikias C.L., Raghuveer M.R. Bispectrum estimation: a digital signal processing framework. Proceedings of the IEEE, volumen 75 (número 7), 1987: 869-891.

NTN SNR. Análisis Vibratorio, 2004 [en línea] [Fecha de consulta: 12 de marzo de 2014]. Disponible en: http://www.ntn-snr. com/industry/es/es-es/index.cfm?page=/industry/home/services/maintenance/analyse_vibratoire

Palomino E. Elementos de medición y análisis de vibraciones mecánica en máquinas rotatorias, Centro de Estudios Ingeniería de Mantenimiento, Publicado por ucv vibraciones mecanicas, 2011 [en línea] [Fecha de consulta: 15 de abril de 2014]. Disponible en: https://es.scribd.com/doc/61342983/Elementos-de-Medicion-y-Analisis-de-Vibraciones-Mecanica-en-Maquinas-Rotatorias

Planeta neutro, 2009 [en línea] [Fecha de consulta: 20 de mayo de 2014]. Disponible en: http://www.renovables-energia.com/ 2009/03/la-energia-eolica-en-el-mundo/

Proakis J.G. y Manolakis D.G. Tratamiento digital de señales, 3a ed., Prentice-Hall, 1998.

Sin M.L., Soong W.L., Ertugrul N. Induction machine on-line condition monitoring and fault diagnosis-a survey, University of Adelaide, 2003, pp. 1-6.

SKF. Fallos en los rodamientos y sus causas [en línea] [Fecha de consulta: 13 de marzo 2014]. Disponible en: http://www.skf.com/ $\mathrm{mx} /$ products/bearings-units-housings/roller-bearings/principles/troubleshooting/bearing-failures-and-their-causes/index.html 
Suarez A. IDEAR. Análisis de fallas en rodamientos, 1998, pp. 1-20.

SKF. Frequency calculator-Bearing frequencies calculation [en línea] [Fecha de consulta: 01 de febrero de 2014]. Disponible en: http://www.skf.com/group/knowledge-centre/engineeringtools/skffrequencycalculator.html

Toledo E., Pinhas I., Aravot D., Akselrod S. Bispectrum and bicoherence for the investigation of very high frecuency peaks in heart rate variability, Proceedings of the IEEE, Computers in Cardiology, Núm. 28. 2001, pp. 667-670.

Villada F. Velilla E., Muñoz N. Protección diferencias de generadores síncronos: Nuevo algoritmo basado en redes neuronales artificiales. editorial académica española, 2012.

Wowk V. Machinery vibration-measurement and analysis, McGraw Hill, 1991.

Ypma A., Upma E., Ligteringen R., Frietman-Eduard E.E., DuinRbert P.W. Recognition of bearing failures using wavelets and neural networks, 1997, pp.1-4.

\begin{abstract}
Este artículo se cita:
Citación estilo Chicago

Medrano-Hurtado, Zulma Yadira, Carlos Pérez-Tello, Julio GómezSarduy, Maximiliano Vera-Pérez. Nueva metodología de diagnóstico de fallas en rodamientos en una máquina síncrona mediante el procesamiento de señales vibro-acústicas empleando análisis de densidad de potencia. Ingeniería Investigación y Tecnología, XVII, 01 (2016): 73-85.
\end{abstract}

\section{Citación estilo ISO 690}

Medrano-Hurtado Z.Y., Pérez-Tello C., Gómez-Sarduy J., VeraPérez M. Nueva metodología de diagnóstico de fallas en rodamientos en una máquina síncrona mediante el procesamiento de señales vibro-acústicas empleando análisis de densidad de potencia. Ingeniería Investigación y Tecnología, volumen XVII (número 1), enero-marzo 2016: 73-85.

\section{Semblanzas de los autores}

Zulma Yadira Medrano-Hurtado. Ingeniera electricista, profesora de la Facultad de Ingeniería de la Universidad Autónoma de Baja California (UABC), en Mexicali Baja California México. Obtuvo la maestria en ingeniería eléctrica en el área de metrología e instrumentación (2007), es estudiante de doctorado en ingeniería eléctrica en el campo de metrología e instrumentación por el Instituto de Ingeniería de la UABC. Sus áreas de interés: metrología e instrumentación, adquisición de datos, procesamiento de señales análisis y diagnóstico de máquinas eléctricas.

Carlos Pérez-Tello. Ingeniero químico del Instituto Tecnológico de Sonora, tiene maestría en ciencias por el Instituto Politécnico Nacional. Obtuvo el doctorado en ciencias por el Instituto Tecnológico de Celaya. Cuenta con experiencia como ingeniero de proceso en plantas metalúrgicas. Trabajó tres años como investigador asociado en el Instituto Mexicano del Petróleo, como investigador en el área de eficiencia energética y ahorro de energía eléctrica, termodinámica aplicada, diseño de experimentos y simulación térmica. Desde 1990 como investigador en el Instituto de Ingeniería de la Universidad Autónoma de Baja California, México. Es autor de varios artículos especializados y coautor de un libro relacionado con inteligencia artificial aplicada a sistemas energéticos.

Julio Rafael Gómez-Sarduy. Ingeniero electricista por la Universidad Central de las Villas (UCLV), Cuba, 1986. Obtuvo su maestría en la misma universidad en 1996. Es doctor en ciencias técnicas con especialidad en máquinas eléctricas por la UCLV (2006). Es profesor titular y actualmente trabaja en el Centro de Estudios de Energía y Medio Ambiente (CEEMA) de la Universidad de Cienfuegos, donde comparte docencia e investigación. Sus temáticas de interés son: máquinas asincrónicas, ahorro en sistemas eléctricos industriales, calidad de la energía e inteligencia artificial para la modelación y el control de sistemas y equipos energéticos. Ha publicado varios artículos y participado en eventos científicos nacionales e internacionales.

Maximiliano Vera-Pérez. Ingeniero mecánico electricista con especialidad en electrónica, obtuvo la maestría y el doctorado en ingeniería electrónica por la Facultad de Ingeniería de la Universidad Autónoma de Baja California (UABC) campus Mexicali. Cuenta con un diplomado de especialidad en docencia y cursos en el desarrollo de cartas descriptivas. Maestro de tiempo completo en la Facultad de Ingeniería de la UABC. En su trayectoria académica y profesional, ha participado y dirigido proyectos educativos y de investigación, ha ocupado el cargo de Coordinador de la carrera de Ingeniero en Electrónica, responsable del Laboratorio de Electrónica y Coordinador del Área de Instrumentación y Control. 